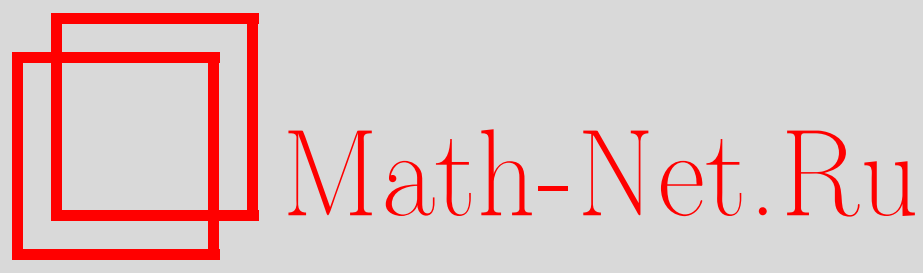

Т. Х. Расулов, Существенный спектр одного модельного оператора, ассоциированного с системой трех частиц на решетке, ТМФ, 2011, том 166, номер 1, 95-109

DOI: https://doi.org/10.4213/tmf6598

Использование Общероссийского математического портала Math-Net.Ru подразумевает, что вы прочитали и согласны с пользовательским соглашением http://www . mathnet.ru/rus/agreement

Параметры загрузки:

IP : 3.89 .197 .203

26 апреля 2023 г., 15:08:39

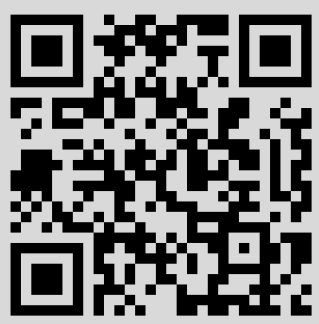




\title{
СУЩЕСТВЕННЫЙ СПЕКТР ОДНОГО МОДЕЛЬНОГО ОПЕРАТОРА, АССОЦИИРОВАННОГО С СИСТЕМОЙ ТРЕХ ЧАСТИЦ НА РЕШЕТКЕ
}

\begin{abstract}
Рассматривается модельный оператор $H$, ассоциированный с системой трех частиц на $\nu$-мерной решетке, взаимодействующих с помощью парных нелокальных потенциалов. Выделяются канальные операторы, и через их спектры описываются местоположение и структура существенного спектра оператора $H$. Для собственных функций оператора $H$ получен аналог уравнения Фаддеева.
\end{abstract}

Ключевые слова: модельный оператор, нелокальный потенциал, класс ГильбертаШмидта, уравнения Фаддеева, существенный спектр, канальный оператор.

\section{1. ВВЕДЕНИЕ}

Исследованию существенного спектра непрерывных и дискретных операторов Шредингера посвящены многие работы (см., например, [1]-[3] и [4]-[7] соответственно). В частности, в работе [5] доказано, что существенный спектр трехчастичного дискретного оператора Шредингера состоит из объединения не более чем конечного числа отрезков даже в том случае, когда соответствующий двухчастичный дискретный оператор Шредингера имеет бесконечное число собственных значений.

Обычно в физической литературе используются "локальные" потенциалы, т. е. операторы умножения на функцию. Однако потенциалы, которые строятся, например, в теории псевдопотенциала [8], оказываются нелокальными и представляют собой, в том числе для периодического оператора, сумму локального потенциала и некоторого конечномерного.

В настоящей работе рассматривается модельный оператор $H$, ассоциированный с системой трех частиц на $\nu$-мерной решетке, взаимодействующих с помощью парных нелокальных потенциалов, где роль двухчастичного дискретного оператора Шредингера играет модель Фридрихса (см., например, работу [6]). Отметим, что оператор $H$ можно рассматривать как некомпактное возмущение оператора, исследованного в работах [6], [7], [9]. Выделены канальные операторы. Местоположение

* Бухарский государственный университет, Бухара, Узбекистан. E-mail: rth@mail.ru

${ }^{\dagger}$ Институт математики университета Берна, Берн, Швейцария.

E-mail: tulkin.rasulov@math.unibe.ch 
и структура существенного спектра оператора $H$ описаны через спектры канальных операторов. Более того, доказано, что существенный спектр этого оператора состоит из объединения не более чем пяти отрезков. Получен аналог уравнения Фаддеева для собственных функций оператора $H$. Отметим, что появление двухчастичных ветвей по обе стороны трехчастичной ветви существенного спектра $H$ (см. ниже теорему 2.3) играет важную роль при изучении конечности или бесконечности частей дискретного спектра, расположенных там, а также на лакунах существенного спектра.

Следует отметить, что двухчастичная и трехчастичная ветви существенного спектра трехчастичного непрерывного оператора Шредингера [1]-[3] представляют собой полубесконечные прямые и пересекаются. В рассматриваемой нами ситуации в отличие от непрерывного случая такие ветви существенного спектра оператора $H$ заполняют отрезки конечной длины, и они могут не пересекаться, т. е. возникает лакуна. Поэтому необходимо изучать ветви существенного спектра по обе стороны трехчастичной ветви, от которой зависит существование двустороннего эффекта Ефимова. В работах [4]-[7], [9] доказано, что рассматриваемые решетчатые операторы не имеют частей существенного и дискретного спектров правее трехчастичной ветви. В этих работах изучение расположения существенного спектра основано на монотонности определителя Фредгольма модели Фридрихса. В данном случае в отличие от предыдущих работ определитель Фредгольма не монотонен, и метод исследования основан на числе собственных значений модели Фридрихса (см. ниже леммы 3.2-3.4).

Структура работы следующая. В разделе 2 модельный оператор рассматривается как ограниченный самосопряженный оператор в гильбертовом пространстве и формулируются основные результаты работы. В разделе 3 , пользуясь разложением в прямой операторный интеграл, изучение спектральных свойств канальных операторов сводится к изучению спектральных свойств модели Фридрихса. В разделе 4 получен аналог уравнения Фаддеева для собственных функций оператора $H$. Раздел 5 посвящен доказательству основных результатов настоящей работы.

\section{2. МОДЕЛЬНЫЙ ОПЕРАТОР И ФОРМУЛИРОВКА ОСНОВНЫХ РЕЗУЛЬТАТОВ}

Пусть $\mathbf{T}^{\nu} \equiv(-\pi ; \pi]^{\nu}-\nu$-мерный куб с соответствующим отождествлением противоположных граней, $L_{2}\left(\mathbf{T}^{\nu}\right)$ и $L_{2}\left(\left(\mathbf{T}^{\nu}\right)^{2}\right)$ - гильбертово пространство квадратичноинтегрируемых (комплекснозначных) функций, определенных на $\mathbf{T}^{\nu}$ и $\left(\mathbf{T}^{\nu}\right)^{2}$ соответственно.

Рассмотрим модельный оператор $H$, действующий в гильбертовом пространстве $L_{2}\left(\left(\mathbf{T}^{\nu}\right)^{2}\right)$ по формуле

$$
H=H_{0}-V_{1}-V_{2},
$$

где операторы $H_{0}$ и $V_{\alpha}, \alpha=1,2$, определяются как

$$
\begin{gathered}
\left(H_{0} f\right)(p, q)=w(p, q) f(p, q) \\
\left(V_{1} f\right)(p, q)=\int v_{1}(q, s) f(p, s) d s, \quad\left(V_{2} f\right)(p, q)=\int v_{2}(p, s) f(s, q) d s .
\end{gathered}
$$


Здесь функция $v_{\alpha}(\cdot, \cdot), \alpha=1,2$, имеет вид

$$
v_{\alpha}(p, q)=v_{\alpha 1}(p) v_{\alpha 1}(q)-v_{\alpha 2}(p) v_{\alpha 2}(q), \quad \alpha=1,2,
$$

a $v_{i j}(\cdot), i, j=1,2$, и $w(\cdot, \cdot)$ - вещественнозначные непрерывные функции на $\mathbf{T}^{\nu}$ и $\left(\mathbf{T}^{\nu}\right)^{2}$ соответственно. Здесь и далее интеграл без указания пределов означает интегрирование по всей области изменения переменных.

При этих предположениях оператор $H$ является ограниченным и самосопряженным в гильбертовом пространстве $L_{2}\left(\left(\mathbf{T}^{\nu}\right)^{2}\right)$.

Отметим, что в работах [10], [11] изучены нелокальные потенциалы с вырожденным ядром вида

$$
\mathcal{V}(p, q)=-\sum_{i=1}^{n} f_{i}(p) g_{i}(q),
$$

рассматриваемые как модели, ассоциированные с системой нескольких частиц, взаимодействующих с помощью парных нелокальных потенциалов. Например, одним из нелокальных потенциалов является потенциал Гаусса, ядро которого для одночастичного случая имеет вид

$$
\mathcal{V}(p, q)=-\mu e^{-\beta\left(p^{2}+q^{2}\right) / 2}, \quad \mu, \beta>0 .
$$

Так как двухчастичные уравнения Шредингера легко разрешимы для нелокальных взаимодействий, их часто используют в ядерной физике и в многочастичных проблемах. Они также систематически используются вместе с уравнениями Фаддеева для систем трех частиц. Основная характеристика этих уравнений состоит в том [11], что частично-волновая $t$-матрица имеет ту же простую форму и может быть продолжена простым способом: наиболее важная характеристика в ядерной физике и в уравнениях Фаддеева.

Положим

$$
w_{1}(p, q)=w(p, q), \quad w_{2}(p, q)=w(q, p) .
$$

Далее предположим, если не оговорено противное, что число $\alpha$ принимает значения 1 и 2.

Обозначим через $\sigma(\cdot), \sigma_{\mathrm{ess}}(\cdot)$ и $\sigma_{\mathrm{disc}}(\cdot)$ соответствено спектр, существенный спектр и дискретный спектр ограниченного самосопряженного оператора.

Для формулировки основных результатов работы наряду с оператором $H$ рассмотрим также ограниченный и самосопряженный оператор $H_{\alpha}$, действующий в гильбертовом пространстве $L_{2}\left(\left(\mathbf{T}^{\nu}\right)^{2}\right)$ по формуле

$$
H_{\alpha}=H_{\alpha}^{0}-V_{\alpha}, \quad\left(H_{\alpha}^{0} f\right)(p, q)=w_{\alpha}(p, q) f(p, q),
$$

и оператор (модель Фридрихса) $h_{\alpha}(p), p \in \mathbf{T}^{\nu}$, действующий в $L_{2}\left(\mathbf{T}^{\nu}\right)$ как

$$
h_{\alpha}(p)=h_{\alpha}^{0}(p)-v_{\alpha},
$$

где операторы $h_{\alpha}^{0}(p), p \in \mathbf{T}^{\nu}$, и $v_{\alpha}$ определяются по правилам

$$
\left(h_{\alpha}^{0}(p) f\right)(q)=w_{\alpha}(p, q) f(q), \quad\left(v_{\alpha} f\right)(q)=\int v_{\alpha}(q, s) f(s) d s .
$$

4 Теоретическая и математическая физика, т. 166, №1, 2011 г. 
Оператор возмущения $v_{\alpha}$ оператора $h_{\alpha}^{0}(p), p \in \mathbf{T}^{\nu}$, является самосопряженным двумерным оператором. Из известной теоремы Вейля [1] о сохранении существенного спектра при возмущениях конечного ранга вытекает, что существенный спектр $\sigma_{\text {ess }}\left(h_{\alpha}(p)\right)$ оператора $h_{\alpha}(p), p \in \mathbf{T}^{\nu}$, совпадает с существенным спектром оператора $h_{\alpha}^{0}(p), p \in \mathbf{T}^{\nu}$. Известно, что $\sigma_{\mathrm{ess}}\left(h_{\alpha}^{0}(p)\right)=\left[m_{\alpha}(p) ; M_{\alpha}(p)\right]$, где числа $m_{\alpha}(p)$ и $M_{\alpha}(p)$ определяются равенствами

$$
m_{\alpha}(p)=\min _{q \in \mathbf{T}^{\nu}} w_{\alpha}(p, q), \quad M_{\alpha}(p)=\max _{q \in \mathbf{T}^{\nu}} w_{\alpha}(p, q)
$$

Из последних двух фактов следует, что $\sigma_{\mathrm{ess}}\left(h_{\alpha}(p)\right)=\left[m_{\alpha}(p) ; M_{\alpha}(p)\right]$.

Пусть $\mathbf{C}$ - комплексная плоскость. При каждом фиксированном $p \in \mathbf{T}^{\nu}$ определим регулярную в $\mathbf{C} \backslash\left[m_{\alpha}(p) ; M_{\alpha}(p)\right]$ функцию

$$
\Delta_{\alpha}(p ; z)=\Delta_{\alpha}^{(1)}(p ; z) \Delta_{\alpha}^{(2)}(p ; z)+\left(\Delta_{\alpha}^{(3)}(p ; z)\right)^{2}
$$

(детерминант Фредгольма, ассоциированный с оператором $h_{\alpha}(p), p \in \mathbf{T}^{\nu}$ ), где

$$
\begin{gathered}
\Delta_{\alpha}^{(1)}(p ; z)=1-\int \frac{v_{\alpha 1}^{2}(s) d s}{w_{\alpha}(p, s)-z}, \quad \Delta_{\alpha}^{(2)}(p ; z)=1+\int \frac{v_{\alpha 2}^{2}(s) d s}{w_{\alpha}(p, s)-z}, \\
\Delta_{\alpha}^{(3)}(p ; z)=\int \frac{v_{\alpha 1}(s) v_{\alpha 2}(s) d s}{w_{\alpha}(p, s)-z} .
\end{gathered}
$$

Тогда имеет место равенство (см. ниже лемму 3.1)

$$
\sigma_{\text {disc }}\left(h_{\alpha}(p)\right)=\left\{z \in \mathbf{C} \backslash\left[m_{\alpha}(p), M_{\alpha}(p)\right]: \Delta_{\alpha}(p ; z)=0\right\}, \quad p \in \mathbf{T}^{\nu} .
$$

Положим

$$
\begin{gathered}
m=\min _{p, q \in \mathbf{T}^{\nu}} w(p, q), \quad M=\max _{p, q \in \mathbf{T}^{\nu}} w(p, q), \\
\sigma_{\mathrm{two}}\left(H_{\alpha}\right)=\bigcup_{p \in \mathbf{T}^{\nu}} \sigma_{\mathrm{disc}}\left(h_{\alpha}(p)\right), \quad \sigma_{\text {three }}\left(H_{\alpha}\right)=[m ; M] .
\end{gathered}
$$

Спектр оператора $H_{\alpha}$ описывается следующей теоремой.

Теорема 2.1. Для спектра оператора $H_{\alpha}$ имеет место равенство

$$
\sigma\left(H_{\alpha}\right)=\sigma_{\text {two }}\left(H_{\alpha}\right) \cup \sigma_{\text {three }}\left(H_{\alpha}\right) \text {. }
$$

Теперь сформулируем результат, который описывает местоположение существенного спектра оператора $H$.

Теорема 2.2. Существенный спектр оператора $H$ совпадает с объединением спектров операторов $H_{1}$ и $H_{2}$, m. е. $\sigma_{\mathrm{ess}}(H)=\sigma\left(H_{1}\right) \cup \sigma\left(H_{2}\right)$. Более того, множество $\sigma_{\mathrm{ess}}(H)$ представляет собой объединение не более чем пяти отрезков.

Очевидно, что операторы $H_{1}$ и $H_{2}$ выбраны единственным образом из оператора $H$ в силу свойства разложимости в прямой операторный интеграл (см. ниже раздел 3). 
Согласно теореме 2.2 операторы $H_{1}$ и $H_{2}$ обладают характеристическим свойством канального оператора соответствующего оператора Шредингера (см., например, работы [4], [5]). По этой причине мы назовем их канальными операторами, соответствующими оператору $H$. Заметим, что канальные операторы $H_{1}$ и $H_{2}$ имеют более простую структуру, чем $H$, и поэтому теорема 2.2 играет важную роль при дальнейших исследованиях спектра оператора $H$.

Положим

$$
\sigma_{\text {two }}(H)=\sigma_{\text {two }}\left(H_{1}\right) \cup \sigma_{\text {two }}\left(H_{2}\right), \quad \sigma_{\text {three }}(H)=\sigma_{\text {three }}\left(H_{1}\right) .
$$

Введем теперь новые подмножества существенного спектра оператора $H$.

ОПРЕДЕЛЕНИЕ. Множества $\sigma_{\text {two }}(H)$ и $\sigma_{\text {three }}(H)$ называются соответственно двухчастичной и трехчастичной ветвями существенного спектра оператора $H$.

Далее предположим, что $\nu=3$, и сформулируем следующее условие для дальнейших рассуждений. Необходимое утверждение приводится в соответствующих размерностях без комментариев.

Основное условиЕ. Функиия $w(\cdot, \cdot)$ имеет невырожденный минимум (максимум) в точках $\left(p_{\min }^{(i)}, q_{\min }^{(i)}\right), i=\overline{1, n}, 1 \leqslant n<\infty\left(\left(p_{\max }^{(j)}, q_{\max }^{(j)}\right), j=\overline{1, m}\right.$, $1 \leqslant m<\infty)$ шестимерного тора.

Если выполняется данное условие, то из непрерывности функции $v_{\alpha i}(\cdot), i=1,2$, на $\mathbf{T}^{3}$ вытекает, что существуют конечные интегралы

$$
\int \frac{v_{\alpha i}(s) v_{\alpha j}(s) d s}{w_{\alpha}(p, s)-m}, \quad \int \frac{v_{\alpha i}(s) v_{\alpha j}(s) d s}{w_{\alpha}(p, s)-M}, \quad i, j=1,2, \quad p \in \mathbf{T}^{3} .
$$

Из теоремы о предельном переходе под знаком интеграла Лебега следует, что при каждом $p \in \mathbf{T}^{3}$ существуют конечные пределы

$$
\lim _{z \rightarrow m-0} \Delta_{\alpha}(p ; z)=\Delta_{\alpha}(p ; m), \quad \lim _{z \rightarrow M+0} \Delta_{\alpha}(p ; z)=\Delta_{\alpha}(p ; M) ;
$$

следовательно, функции $\Delta_{\alpha}(\cdot ; m)$ и $\Delta_{\alpha}(\cdot ; M)$ являются непрерывными на $\mathbf{T}^{3}$.

Обозначим

$$
\begin{array}{rlrl}
a_{\alpha} & =\min \left\{\sigma_{\mathrm{two}}\left(H_{\alpha}\right) \cap(-\infty ; m]\right\}, & b_{\alpha}=\max \left\{\sigma_{\mathrm{two}}\left(H_{\alpha}\right) \cap(-\infty ; m]\right\}, \\
c_{\alpha}=\min \left\{\sigma_{\mathrm{two}}\left(H_{\alpha}\right) \cap[M ;+\infty)\right\}, & d_{\alpha}=\max \left\{\sigma_{\mathrm{two}}\left(H_{\alpha}\right) \cap[M ;+\infty)\right\} .
\end{array}
$$

Следующая теорема описывает структуру спектра оператора $H_{\alpha}$.

Теорема 2.3. Пусть выполняется основное условие. Предположим, что:

1) $\min _{p \in \mathbf{T}^{3}} \Delta_{\alpha}(p ; m) \geqslant 0 ;$ тогда:
а) $\sigma\left(H_{\alpha}\right)=[m ; M]$, если $\min _{p \in \mathbf{T}^{3}} \Delta_{\alpha}(p ; M) \geqslant 0$;
б) $\sigma\left(H_{\alpha}\right)=[m ; M] \cup\left[c_{\alpha} ; d_{\alpha}\right], c_{\alpha}>M$, если $\max _{p \in \mathbf{T}^{3}} \Delta_{\alpha}(p ; M)<0$;

2) $\max _{p \in \mathbf{T}^{3}} \Delta_{\alpha}(p ; m)<0 ;$ тогда:

a) $\sigma\left(H_{\alpha}\right)=\left[a_{\alpha} ; b_{\alpha}\right] \cup[m ; M], b_{\alpha}<m$, если $\min _{p \in \mathbf{T}^{3}} \Delta_{\alpha}(p ; M) \geqslant 0$;

б) $\sigma\left(H_{\alpha}\right)=\left[a_{\alpha} ; b_{\alpha}\right] \cup[m ; M] \cup\left[c_{\alpha} ; d_{\alpha}\right], b_{\alpha}<m u c_{\alpha}>M$, если $\max _{p \in \mathbf{T}^{3}} \Delta_{\alpha}(p ; M)<0$. 
Отметим, что теоремы 2.2 и 2.3 вместе описывают местоположение и структуру существенного спектра.

ЗАмЕчАниЕ 2.1. Класс функций $v_{i j}(\cdot), i, j=1,2$, и $w(\cdot, \cdot)$, удовлетворяющих условиям теоремы 2.3, является непустым (см. ниже лемму 3.5 ).

\section{3. НЕКОТОРЫЕ СПЕКТРАЛЬНЫЕ СВОЙСТВА ОПЕРАТОРОВ $H_{\alpha}$ И $H_{\alpha}(p), p \in \mathbf{T}^{\nu}$}

В этом разделе исследование спектральных свойств оператора канала $H_{\alpha}$ с использованием разложения в прямой операторный интеграл сводится к изучению спектральных свойств модели Фридрихса $h_{\alpha}(p), p \in \mathbf{T}^{\nu}$, которая определена формулой (2.1). Описывается дискретный спектр оператора $h_{\alpha}(p), p \in \mathbf{T}^{\nu}$.

Можно легко проверить, что оператор $H_{1}\left(H_{2}\right)$ коммутирует с любым оператором умножения на ограниченную функцию $u_{1}(p)\left(u_{2}(q)\right)$ в $L_{2}\left(\left(\mathbf{T}^{\nu}\right)^{2}\right)$.

Из разложения в прямой интеграл пространства $L_{2}\left(\left(\mathbf{T}^{\nu}\right)^{2}\right)$

$$
L_{2}\left(\left(\mathbf{T}^{\nu}\right)^{2}\right)=\int \oplus L_{2}\left(\mathbf{T}^{\nu}\right) d p
$$

следует, что оператор $H_{\alpha}$ разлагается в прямой операторной интеграл

$$
H_{\alpha}=\int \oplus h_{\alpha}(p) d p
$$

где оператор $h_{\alpha}(p), p \in \mathbf{T}^{\nu}$, определен формулой (2.1). Отметим, что в разложении (3.1) под знаком прямого интеграла стоят одинаковые слои.

Установим связь между собственными значениями оператора $h_{\alpha}(p), p \in \mathbf{T}^{\nu}$, и нулями функции $\Delta_{\alpha}(p ; \cdot)$. Верна следующая лемма.

ЛЕмма 3.1. При каждом фиксированном $p \in \mathbf{T}^{\nu}$ число $z \in \mathbf{C} \backslash \sigma_{\mathrm{ess}}\left(h_{\alpha}(p)\right)$ является собственным значением оператора $h_{\alpha}(p)$ тогда и только тогда, когда $\Delta_{\alpha}(p ; z)=0$.

ДокАЗАтеЛьство. Пусть число $z \in \mathbf{C} \backslash \sigma_{\mathrm{ess}}\left(h_{\alpha}(p)\right)$ - собственное значение оператора $h_{\alpha}(p), p \in \mathbf{T}^{\nu}$, и пусть $f \in L_{2}\left(\mathbf{T}^{\nu}\right)$ - соответствующая собственная функция, т. е., уравнение

$$
w_{\alpha}(p, q) f(q)-\int\left[v_{\alpha 1}(q) v_{\alpha 1}(s)-v_{\alpha 2}(q) v_{\alpha 2}(s)\right] f(s) d s=z f(q)
$$

имеет ненулевое решение $f \in L_{2}\left(\mathbf{T}^{\nu}\right)$.

Заметим, что для любых $z \in \mathbf{C} \backslash \sigma_{\mathrm{ess}}\left(h_{\alpha}(p)\right)$ и $q \in \mathbf{T}^{\nu}$ имеет место соотношение $w_{\alpha}(p, q)-z \neq 0, p \in \mathbf{T}^{\nu}$. Тогда из уравнения (3.2) для $f$ имеем

$$
f(q)=\frac{v_{\alpha 1}(q) C_{\alpha}^{(1)}-v_{\alpha 2}(q) C_{\alpha}^{(2)}}{w_{\alpha}(p, q)-z},
$$

где

$$
C_{\alpha}^{(i)}=\int v_{\alpha i}(s) f(s) d s, \quad i=1,2 .
$$


Подставляя выражение (3.3) в равенство (3.4) получим, что уравнение (3.2) имеет ненулевое решение тогда и только тогда, когда система уравнений

$$
\begin{aligned}
\left(1-\int \frac{v_{\alpha 1}^{2}(s) d s}{w_{\alpha}(p, s)-z}\right) C_{\alpha}^{(1)}+\int \frac{v_{\alpha 1}(s) v_{\alpha 2}(s) d s}{w_{\alpha}(p, s)-z} C_{\alpha}^{(2)}=0, \\
-\int \frac{v_{\alpha 1}(s) v_{\alpha 2}(s) d s}{w_{\alpha}(p, s)-z} C_{\alpha}^{(1)}+\left(1+\int \frac{v_{\alpha 2}^{2}(s) d s}{w_{\alpha}(p, s)-z}\right) C_{\alpha}^{(2)}=0
\end{aligned}
$$

имеет ненулевое решение $\left(C_{\alpha}^{(1)}, C_{\alpha}^{(2)}\right) \in \mathbf{C}^{2}$, т. е. когда $\Delta_{\alpha}(p ; z)=0$, где $\mathbf{C}^{2}$ - декартов квадрат множества С. Лемма доказана.

Для любого ограниченного самосопряженного оператора $A$, действующего в гильбертовом пространстве $\mathcal{H}$, обозначим через $\mathcal{H}_{A}(z)$ такое подпространство, что $(A f, f)<\lambda\|f\|$ для любого $f \in \mathcal{H}_{A}(z)$, и положим

$$
N(\lambda, A)=\sup _{\mathcal{H}_{A}(z)} \operatorname{dim} \mathcal{H}_{A}(z)
$$

Число $N(\lambda, A)$ равно бесконечности, если $\lambda>\min \sigma_{\text {ess }}(A)$; если число $N(\lambda, A)$ конечно, то оно равно числу собственных значений оператора $A$, меньших $\lambda$, с учетом кратности.

Лемма 3.2. Для любого $p \in \mathbf{T}^{\nu}$ оператор $h_{\alpha}(p)$ может иметь не более одного простого собственного значения, лежащего левее $m_{\alpha}(p)$ и правее $M_{\alpha}(p)$.

ДоказАтельство. Введем оператор $v_{\alpha i}, i=1,2$, действующий в $L_{2}\left(\mathbf{T}^{\nu}\right)$ как

$$
\left(v_{\alpha i} f\right)(q)=v_{\alpha i}(q) \int v_{\alpha i}(s) f(s) d s, \quad i=1,2 .
$$

Тогда оператор $v_{\alpha}$ можно записать в виде $v_{\alpha}=v_{\alpha 1}-v_{\alpha 2}$. Положим $h_{\alpha}^{(1)}=h_{\alpha}^{0}-v_{\alpha 1}$. Так как оператор $v_{\alpha i}, i=1,2$, неотрицательный, можно легко показать, что $h_{\alpha}(p) \geqslant$ $h_{\alpha}^{(1)}(p)$ и, следовательно,

$$
\mathcal{H}_{h_{\alpha}(p)}(z) \subset \mathcal{H}_{h_{\alpha}^{(1)}(p)}(z), \quad z \leqslant m_{\alpha}(p)
$$

Это означает, что

$$
N\left(z, h_{\alpha}(p)\right) \leqslant N\left(z, h_{\alpha}^{(1)}(p)\right), \quad z \leqslant m_{\alpha}(p) .
$$

Так как определитель Фредгольма $\Delta_{\alpha}^{(1)}(p ; \cdot)$ оператора $h_{\alpha}^{(1)}(p)$ монотонно убывает на полуоси $\left(-\infty, m_{\alpha}(p)\right)$, то $N\left(m_{\alpha}(p), h_{\alpha}^{(1)}(p)\right) \leqslant 1$; следовательно, в силу неравенства $(3.5)$ верно $N\left(m_{\alpha}(p), h_{\alpha}(p)\right) \leqslant 1$. Это означает, что для любого $p \in \mathbf{T}^{\nu}$ оператор $h_{\alpha}(p)$ может иметь не более чем одно простое собственное значение, лежащее левее $m_{\alpha}(p)$.

Аналогично можно показать, что $N\left(-M_{\alpha}(p),-h_{\alpha}(p)\right) \leqslant 1$. Лемма доказана.

Следующая лемма описывает множество собственных значений оператора $h_{\alpha}(p)$, $p \in \mathbf{T}^{3}$, на $(-\infty, m)$. 
ЛЕмма 3.3. Пусть выполняется основное условие. Тогда:

1) если $\min _{p \in \mathbf{T}^{3}} \Delta_{\alpha}(p ; m) \geqslant 0$, то для любого $p \in \mathbf{T}^{3}$ оператор $h_{\alpha}(p)$ не имеет собственных значений на $(-\infty, m)$;

2) если $\max _{p \in \mathbf{T}^{3}} \Delta_{\alpha}(p ; m)<0$, то для любого $p \in \mathbf{T}^{3}$ оператор $h_{\alpha}(p)$ имеет единственное простое собственное значение на $(-\infty, m)$.

Лемма 3.3 доказывается аналогично следующей лемме, которая описывает множество собственных значений оператора $h_{\alpha}(p), p \in \mathbf{T}^{3}$, на $(M,+\infty)$.

Лемма 3.4. Пусть выполняется основное условие. Тогда:

1) если $\min _{p \in \mathbf{T}^{3}} \Delta_{\alpha}(p ; M) \geqslant 0$, то для любого $p \in \mathbf{T}^{3}$ оператор $h_{\alpha}(p)$ не имеет собственных значений на $(M,+\infty)$;

2) если $\max _{p \in \mathbf{T}^{3}} \Delta_{\alpha}(p ; M)<0$, то для любого $p \in \mathbf{T}^{3}$ оператор $h_{\alpha}(p)$ имеет единственное простое собственное значение на $(M,+\infty)$.

ДоказАтельство. 1) Пусть $\min _{p \in \mathbf{T}^{3}} \Delta_{\alpha}(p ; M) \geqslant 0$. Тогда для любого $p \in \mathbf{T}^{3}$ имеет место неравенство $\Delta_{\alpha}(p ; M) \geqslant 0$. Из непрерывности функции $\Delta_{\alpha}(p ; \cdot)$ на $[M, \infty)$ и равенства

$$
\lim _{z \rightarrow+\infty} \Delta_{\alpha}(p ; z)=1
$$

следует, что функция $\Delta_{\alpha}(p ; \cdot)$ не имеет нулей на $(M, \infty)$ или имеет по крайней мере два (с учетом кратности) нуля на $(M, \infty)$. Во втором случае по лемме 3.1 оператор $h_{\alpha}(p)$ имеет по крайней мере два (с учетом кратности) собственных значения на $(M, \infty)$. Это противоречит утверждению леммы 3.2. Таким образом, для любого $p \in \mathbf{T}^{3}$ оператор $h_{\alpha}(p)$ не имеет собственных значений на $(M, \infty)$. Здесь мы использовали также тот факт, что число $z_{0} \in \mathbf{C} \backslash \sigma_{\mathrm{ess}}\left(h_{\alpha}(p)\right)$ является $n$-кратным собственным значением $h_{\alpha}(p)$ тогда и только тогда, когда число $z_{0}$ является $n$-кратным нулем функции $\Delta_{\alpha}(p ; \cdot)[12]$.

2) Пусть $\max _{p \in \mathbf{T}^{3}} \Delta_{\alpha}(p ; M)<0$. Тогда для любого $p \in \mathbf{T}^{3}$ имеет место неравенство $\Delta_{\alpha}(p ; M)<0$. В силу равенства (3.6) функция $\Delta_{\alpha}(p ; \cdot)$ при всех $p \in \mathbf{T}^{3}$ имеет единственный простой нуль на $(M, \infty)$ или имеет по крайней мере три (с учетом кратности) нуля на $(M, \infty)$. Рассуждая аналогично предыдущему, получаем, что возможен только первый случай, который в силу леммы 3.1 означает, что при всех $p \in \mathbf{T}^{3}$ оператор $h_{\alpha}(p)$ имеет единственное простое собственное значение на $(M,+\infty)$. Лемма 3.4 доказана.

ЛЕмма 3.5. Пусть $w(\cdot, \cdot)$ - любая функиия, удовлетворяющая основному условию. Предположим, что $v_{\alpha i}(p)=\sqrt{\mu_{\alpha i}} \hat{v}_{\alpha i}(p), \mu_{\alpha i}>0, i=1,2$, такое, что функция $\hat{v}_{\alpha i}(\cdot), i=1,2$, непрерьвна на $\mathbf{T}^{3}$ с компактным носителем $\Omega_{\alpha i} \subset \mathbf{T}^{3}$ $u \Omega_{\alpha 1} \cap \Omega_{\alpha 2}=\varnothing$.

Положим

$$
\begin{array}{ll}
\mu_{\alpha 1}^{(1)}=\min _{p \in \mathbf{T}^{3}}\left(\int_{\Omega_{\alpha 1}} \frac{\hat{v}_{\alpha 1}^{2}(s) d s}{w_{\alpha}(p, s)-m}\right)^{-1}, & \mu_{\alpha 1}^{(2)}=\max _{p \in \mathbf{T}^{3}}\left(\int_{\Omega_{\alpha 1}} \frac{\hat{v}_{\alpha 1}^{2}(s) d s}{w_{\alpha}(p, s)-m}\right)^{-1}, \\
\mu_{\alpha 2}^{(1)}=\min _{p \in \mathbf{T}^{3}}\left(\int_{\Omega_{\alpha 2}} \frac{\hat{v}_{\alpha 2}^{2}(s) d s}{M-w_{\alpha}(p, s)}\right)^{-1}, & \mu_{\alpha 2}^{(2)}=\max _{p \in \mathbf{T}^{3}}\left(\int_{\Omega_{\alpha 2}} \frac{\hat{v}_{\alpha 2}^{2}(s) d s}{M-w_{\alpha}(p, s)}\right)^{-1} .
\end{array}
$$


Рассмотрим случаи:
a) $0<\mu_{\alpha 1} \leqslant \mu_{\alpha 1}^{(1)}, 0<\mu_{\alpha 2} \leqslant \mu_{\alpha 2}^{(1)}$,
б) $0<\mu_{\alpha 1} \leqslant \mu_{\alpha 1}^{(1)}, \mu_{\alpha 2}>\mu_{\alpha 2}^{(2)}$,
в) $\mu_{\alpha 1}>\mu_{\alpha 1}^{(2)}, 0<\mu_{\alpha 2} \leqslant \mu_{\alpha 2}^{(1)}$,
г) $\mu_{\alpha 1}>\mu_{\alpha 1}^{(2)}, \mu_{\alpha 2}>\mu_{\alpha 2}^{(2)}$.

Если выполняются условия "а", "б", "в", "г", то выполняются и условия 1а, 1б, 2а, 26 в утверждении теоремы 2.3.

ЗАмЕчАниЕ 3.1. Отметим, что примерами рассмотренных в лемме 3.5 функций $w(\cdot, \cdot)$ и $\hat{v}_{\alpha i}(\cdot), i=1,2$, являются следующие:

$$
\begin{gathered}
w(p, q)=\varepsilon(p)+4 \varepsilon\left(\frac{1}{2}(p+q)\right)+\varepsilon(q), \quad \varepsilon(p)=\sum_{i=1}^{3}\left(1-\cos p^{(i)}\right), \\
\hat{v}_{\alpha 1}(p)=\left\{\begin{array}{lll}
\alpha \sum_{i=1}^{3}\left(\cos p^{(i)}-\cos 1\right), & \text { если } & p \in B_{1}(0), \\
0, & \text { если } & p \in \mathbf{T}^{3} \backslash B_{1}(0),
\end{array}\right. \\
\hat{v}_{\alpha 2}(p)=\left\{\begin{array}{lll}
\alpha \sum_{i=1}^{3}\left(\cos p^{(i)}+\cos 1\right), & \text { если } & p \in B_{1}(\bar{\pi}), \\
0, & \text { если } & p \in \mathbf{T}^{3} \backslash B_{1}(\bar{\pi}) .
\end{array}\right.
\end{gathered}
$$

Здесь $B_{1}\left(p_{0}\right)=\left\{p \in \mathbf{T}^{3}:\left|p-p_{0}\right| \leqslant 1\right\}, p_{0} \in \mathbf{T}^{3}$. Видно, что функция $w(\cdot, \cdot)$ имеет единственный невырожденный минимум в точке $(0,0) \in\left(\mathbf{T}^{3}\right)^{2}$ и максимум в точке $(\bar{\pi}, \bar{\pi}) \in\left(\mathbf{T}^{3}\right)^{2}, \bar{\pi}=(\pi, \pi, \pi) \in \mathbf{T}^{3}$, т. е. $n=m=1$.

ДокАЗАТЕЛЬСтво ЛЕммы 3.5. Сначала отметим, что при наложенных на функции $\hat{v}_{\alpha i}(\cdot), i=1,2$, условиях имеет место равенство $\Delta_{\alpha}(p ; z)=\Delta_{\alpha}^{(1)}(p ; z) \Delta_{\alpha}^{(2)}(p ; z)$. Докажем лемму для случая (в); остальные утверждения доказываются аналогично.

Пусть $\mu_{\alpha 1}>\mu_{\alpha 1}^{(2)}, 0<\mu_{\alpha 2} \leqslant \mu_{\alpha 2}^{(1)}$. Тогда

$$
\max _{p \in \mathbf{T}^{3}} \Delta_{\alpha}(p ; m) \leqslant \max _{p \in \mathbf{T}^{3}}\left(1-\mu_{\alpha 1} \int_{\Omega_{\alpha 1}} \frac{\hat{v}_{\alpha 1}^{2}(s) d s}{w_{\alpha}(p, s)-m}\right) \max _{p \in \mathbf{T}^{3}}\left(1+\mu_{\alpha 2} \int_{\Omega_{\alpha 2}} \frac{\hat{v}_{\alpha 2}^{2}(s) d s}{w_{\alpha}(p, s)-m}\right) .
$$

В последнем неравенстве второй множитель, стоящий в правой части, положителен при любом $\mu_{\alpha 2}>0$. Так как $\mu_{\alpha 1}>\mu_{\alpha 1}^{(2)}$, то первый множитель отрицателен, т. е. $\max _{p \in \mathbf{T}^{3}} \Delta_{\alpha}(p ; m)<0$. Точно так же показывается, что при любых $\mu_{\alpha 1}>0$ и $0<\mu_{\alpha 2} \leqslant \mu_{\alpha 2}^{(1)}$ имеет место $\min _{p \in \mathbf{T}^{3}} \Delta_{\alpha}(p ; M) \geqslant 0$. Лемма доказана.

\section{4. АНАЛОГ УРАВНЕНИЯ ФАДДЕЕВА ДЛЯ СОБСТВЕННЫХ ФУНКЦИЙ ОПЕРАТОРА $H$}

В этом разделе мы получим аналог системы интегральных уравнений Фаддеева для собственных функций оператора $H$, который играет важную роль при исследовании спектра этого оператора. Часть наших построений и рассуждений аналогичны тем, что содержатся в работе [13], и в этих случаях мы ограничиваемся соответствующей ссылкой. 
Пусть $L_{2}^{(2)}\left(\mathbf{T}^{\nu}\right)$ - гильбертово пространство двухкомпонентных вектор-функций $f=\left(f_{1}, f_{2}\right), f_{\alpha} \in L_{2}\left(\mathbf{T}^{\nu}\right), \alpha=1,2$. При каждом $z \in \mathbf{C} \backslash \sigma_{\text {three }}(H)$ вводим блочно-операторные матрицы $A(z)$ и $K(z)$, действующие в пространстве $L_{2}^{(4)}\left(\mathbf{T}^{\nu}\right)=L_{2}^{(2)}\left(\mathbf{T}^{\nu}\right) \oplus$ $L_{2}^{(2)}\left(\mathbf{T}^{\nu}\right)$ по формулам

$$
A(z)=\left(\begin{array}{cc}
A_{1}(z) & 0 \\
0 & A_{2}(z)
\end{array}\right), \quad K(z)=\left(\begin{array}{cc}
0 & K_{2}(z) \\
K_{1}(z) & 0
\end{array}\right)
$$

где матричные элементы $A_{\alpha}(z)$ и $K_{\alpha}(z)$ также являются блочно-операторными матрицами, действующими в пространстве $L_{2}^{(2)}\left(\mathbf{T}^{\nu}\right)$ :

$$
A_{\alpha}(z)=\left(\begin{array}{cc}
A_{11}^{(\alpha)}(z) & A_{12}^{(\alpha)}(z) \\
A_{21}^{(\alpha)}(z) & A_{22}^{(\alpha)}(z)
\end{array}\right), \quad K_{\alpha}(z)=\left(\begin{array}{cc}
K_{11}^{(\alpha)}(z) & K_{12}^{(\alpha)}(z) \\
K_{21}^{(\alpha)}(z) & K_{22}^{(\alpha)}(z)
\end{array}\right) .
$$

Здесь $A_{i j}^{(\alpha)}(z): L_{2}\left(\mathbf{T}^{\nu}\right) \rightarrow L_{2}\left(\mathbf{T}^{\nu}\right), i, j=1,2, z \in \mathbf{C} \backslash \sigma_{\text {three }}(H)$, - оператор умножения на функцию $a_{i j}^{(\alpha)}(p ; z)$ :

$$
\begin{gathered}
a_{11}^{(\alpha)}(p ; z)=\Delta_{\alpha}^{(1)}(p ; z), \quad a_{12}^{(\alpha)}(p ; z)=-a_{21}^{(\alpha)}(p ; z)=\Delta_{\alpha}^{(3)}(p ; z), \\
a_{22}^{(\alpha)}(p ; z)=\Delta_{\alpha}^{(2)}(p ; z),
\end{gathered}
$$

а операторы $K_{i j}^{(\alpha)}(z): L_{2}\left(\mathbf{T}^{\nu}\right) \rightarrow L_{2}\left(\mathbf{T}^{\nu}\right), i, j=1,2, z \in \mathbf{C} \backslash \sigma_{\text {three }}(H),-$ интегральные операторы с ядрами $K_{i j}^{(\alpha)}(p, s ; z)(s-$ переменная интегрирования):

$$
\begin{aligned}
& K_{11}^{(1)}(p, s ; z)=K_{11}^{(2)}(s, p ; z)=\frac{v_{11}(p) v_{21}(s)}{w_{2}(p, s)-z}, \\
& K_{12}^{(1)}(p, s ; z)=-K_{21}^{(2)}(s, p ; z)=-\frac{v_{12}(s) v_{21}(q)}{w_{2}(p, s)-z}, \\
& K_{21}^{(1)}(p, s ; z)=-K_{12}^{(1)}(s, p ; z)=\frac{v_{11}(s) v_{22}(q)}{w_{2}(p, s)-z}, \\
& K_{22}^{(1)}(p, s ; z)=K_{22}^{(2)}(s, p ; z)=-\frac{v_{12}(p) v_{22}(s)}{w_{2}(p, s)-z} .
\end{aligned}
$$

Заметим, что при каждом $z \in \mathbf{C} \backslash \sigma_{\text {three }}(H)$ операторы $K_{i j}^{(\alpha)}(z), i, j=1,2$, принадлежат классу Гильберта-Шмидта, следовательно, $K(z)$ является компактным оператором.

Лемма 4.1. При каждом $z \in \mathbf{C} \backslash\left(\sigma_{\text {two }}(H) \cup \sigma_{\text {three }}(H)\right)$ оператор $A(z)$ является ограниченным и обратимым; обратный оператор $A^{-1}(z)$ определен формулой

$$
A^{-1}(z)=\left(\begin{array}{cc}
A_{1}^{-1}(z) & 0 \\
0 & A_{2}^{-1}(z)
\end{array}\right), \quad \text { əде } \quad A_{\alpha}^{-1}(z)=\left(\begin{array}{cc}
B_{11}^{(\alpha)}(z) & B_{12}^{(\alpha)}(z) \\
B_{21}^{(\alpha)}(z) & B_{22}^{(\alpha)}(z)
\end{array}\right) .
$$


Здесъ $B_{i j}^{(\alpha)}(z): L_{2}\left(\mathbf{T}^{\nu}\right) \rightarrow L_{2}\left(\mathbf{T}^{\nu}\right), i, j=1,2, z \in \mathbf{C} \backslash\left(\sigma_{\text {two }}(H) \cup \sigma_{\text {three }}(H)\right)$ - onepamop умножения на функцию $b_{i j}^{(\alpha)}(p ; z)$ :

$$
\begin{aligned}
b_{11}^{(\alpha)}(p ; z) & =\frac{a_{22}^{(\alpha)}(p ; z)}{\Delta_{\alpha}(p ; z)}, & b_{12}^{(\alpha)}(p ; z) & =-\frac{a_{12}^{(\alpha)}(p ; z)}{\Delta_{\alpha}(p ; z)}, \\
b_{21}^{(\alpha)}(p ; z) & =-\frac{a_{21}^{(\alpha)}(p ; z)}{\Delta_{\alpha}(p ; z)}, & b_{22}^{(\alpha)}(p ; z) & =\frac{a_{11}^{(\alpha)}(p ; z)}{\Delta_{\alpha}(p ; z)} .
\end{aligned}
$$

Лемма 4.1 доказывается аналогично соответствующей лемме из работы [13].

Так как при каждом $z \in \mathbf{C} \backslash\left(\sigma_{\text {two }}(H) \cup \sigma_{\text {three }}(H)\right)$ оператор $A(z)$ обратим, то для таких $z$ мы определим оператор вида

$$
T(z)=A^{-1}(z) K(z)=\left(\begin{array}{cc}
0 & A_{1}^{-1}(z) K_{2}(z) \\
A_{2}^{-1}(z) K_{1}(z) & 0
\end{array}\right)
$$

Следующая лемма устанавливает связь между собственными значениями операторов $H$ и $T(z)$.

Лемма 4.2. Число $z \in \mathbf{C} \backslash\left(\sigma_{\text {two }}(H) \cup \sigma_{\text {three }}(H)\right)$ является собственным значением оператора $H$ тогда и только тогда, когда оператор $T(z)$ имеет собственное значение, равное единице, и их кратности совпадают.

ДоказАтельство. Пусть $z \in \mathbf{C} \backslash\left(\sigma_{\text {two }}(H) \cup \sigma_{\text {three }}(H)\right)$ - собственное значение оператора $H$ и $f \in L_{2}\left(\left(\mathbf{T}^{\nu}\right)^{2}\right)$ - соответствующая собственная функция, т. е. уравнение $H f=z f$, или уравнение

$$
\begin{gathered}
(w(p, q)-z) f(p, q)-\int\left[v_{11}(q) v_{11}(s)-v_{12}(q) v_{12}(s)\right] f(p, s) d s- \\
-\int\left[v_{21}(p) v_{21}(s)-v_{22}(p) v_{22}(s)\right] f(s, q) d s=0
\end{gathered}
$$

имеет нетривиальное решение $f \in L_{2}\left(\left(\mathbf{T}^{\nu}\right)^{2}\right)$.

Так как $z \notin \sigma_{\text {three }}(H)$, для $f$ из уравнения (4.1) имеем

$$
f(p, q)=\frac{v_{11}(q) g_{1}^{(1)}(p)-v_{12}(q) g_{2}^{(1)}(p)+v_{21}(p) g_{1}^{(2)}(q)-v_{22}(p) g_{2}^{(2)}(q)}{w(p, q)-z}
$$

где

$$
g_{\alpha}^{(1)}(p)=\int v_{1 \alpha}(s) f(p, s) d s, \quad g_{\alpha}^{(2)}(p)=\int v_{2 \alpha}(s) f(s, p) d s .
$$


Подставляя выражение (4.2) в равенство (4.3), получим, что система уравнений

$$
\begin{aligned}
& \Delta_{1}^{(1)}(p ; z) g_{1}^{(1)}(p)+\Delta_{3}^{(1)}(p ; z) g_{2}^{(1)}(p)= \\
& \quad=v_{21}(p) \int \frac{v_{11}(s) g_{1}^{(2)}(s)}{w_{1}(p, s)-z} d s-v_{22}(p) \int \frac{v_{11}(s) g_{2}^{(2)}(s)}{w_{1}(p, s)-z} d s, \\
& -\Delta_{3}^{(1)}(p ; z) g_{1}^{(1)}(p)+\Delta_{2}^{(1)}(p ; z) g_{2}^{(1)}(p)= \\
& \quad=v_{21}(p) \int \frac{v_{12}(s) g_{1}^{(2)}(s)}{w_{1}(p, s)-z} d s-v_{22}(p) \int \frac{v_{12}(s) g_{2}^{(2)}(s)}{w_{1}(p, s)-z} d s, \\
& \Delta_{1}^{(2)}(p ; z) g_{1}^{(2)}(p)+\Delta_{3}^{(2)}(p ; z) g_{2}^{(2)}(p)= \\
& \quad=v_{11}(p) \int \frac{v_{21}(s) g_{1}^{(1)}(s)}{w_{2}(p, s)-z} d s-v_{12}(p) \int \frac{v_{21}(s) g_{2}^{(1)}(s)}{w_{2}(p, s)-z} d s, \\
& -\Delta_{3}^{(2)}(p ; z) g_{1}^{(2)}(p)+\Delta_{2}^{(2)}(p ; z) g_{2}^{(2)}(p)= \\
& \quad=v_{11}(p) \int \frac{v_{22}(s) g_{1}^{(1)}(s)}{w_{2}(p, s)-z} d s-v_{12}(p) \int \frac{v_{22}(s) g_{2}^{(1)}(s)}{w_{2}(p, s)-z} d s,
\end{aligned}
$$

или уравнение

$$
A(z) g=K(z) g, \quad g=\left(g_{1}^{(1)}, g_{2}^{(1)}, g_{1}^{(2)}, g_{2}^{(2)}\right) \in L_{2}^{(4)}\left(\mathbf{T}^{\nu}\right)
$$

имеет нетривиальное решение тогда и только тогда, когда уравнение (4.1) имеет нетривиальное решение и линейные подпространства, порожденные решениями уравнений (4.1) и (4.4), имеют одинаковые размерности.

По лемме 4.1 при каждом $z \in \mathbf{C} \backslash\left(\sigma_{\text {two }}(H) \cup \sigma_{\text {three }}(H)\right)$ оператор $A(z)$ обратим; следовательно, уравнение $g=A^{-1}(z) K(z) g$, т. е. уравнение $g=T(z) g$, имеет нетривиальное решение тогда и только тогда, когда уравнение (4.4) имеет нетривиальное решение. Здесь также линейные подпространства, порожденные решениями уравнений (4.4) и $g=T(z) g$, имеют одинаковые размерности. Лемма доказана.

ЗАмЕчАниЕ 4.1. Заметим, что уравнение $T(z) g=g$ обычно называется аналогом уравнения Фаддеева для собственных функций оператора $H$.

Видно, что при каждом $z<\min \left(\sigma_{\text {two }}(H) \cup \sigma_{\text {three }}(H)\right)$ оператор $A(z)$ является положительным и, следовательно, существует его положительный квадратный корень $A^{-1 / 2}(z)$. Для таких $z$ определим оператор $\widehat{T}(z)=A^{-1 / 2}(z) K(z) A^{-1 / 2}(z)$, являющийся симметризованным вариантом уравнения Фаддеева для собственных функций оператора $H$.

Следующая лемма доказывается аналогично лемме 4.2 и устанавливает связь между собственными значениями операторов $H$ и $\widehat{T}(z)$.

Лемма 4.3. Число $z<\min \left(\sigma_{\mathrm{two}}(H) \cup \sigma_{\text {three }}(H)\right)$ является собственным значением оператора $H$ тогда и только тогда, когда оператор $\widehat{T}(z)$ имеет собственное значение, равное единице, и их кратности совпадают. 


\section{5. ДОКАЗАТЕЛЬСТВО ОСНОВНЫХ РЕЗУЛЬТАТОВ}

В этом разделе, пользуясь утверждениями разделов 3 и 4, а также критерием Вейля и теоремой о спектре разложимых операторов, мы докажем основные результаты работы.

ДокАЗАТЕЛЬСТво тЕОРЕмы 2.1. Применяя теорему о спектре разложимых операторов и учитывая равенства

$$
\sigma\left(h_{\alpha}(p)\right)=\sigma_{\text {disc }}\left(h_{\alpha}(p)\right) \cup\left[m_{\alpha}(p) ; M_{\alpha}(p)\right], \quad \bigcup_{p \in \mathbf{T}^{\nu}}\left[m_{\alpha}(p) ; M_{\alpha}(p)\right]=[m ; M],
$$

мы приходим к утверждению теоремы 2.1.

ДоказАТЕЛЬСтво теоремы 2.2. Сначала докажем, что $\sigma\left(H_{1}\right) \cup \sigma\left(H_{2}\right) \subset \sigma_{\text {ess }}(H)$. С этой целью множество $\sigma\left(H_{1}\right) \cup \sigma\left(H_{2}\right)$ перепишем в виде

$$
\sigma\left(H_{1}\right) \cup \sigma\left(H_{2}\right)=\sigma_{\text {two }}\left(H_{1}\right) \cup \sigma_{\text {two }}\left(H_{2}\right) \cup \sigma_{\text {three }}\left(H_{1}\right) \text {. }
$$

Включение $\sigma_{\text {three }}\left(H_{1}\right) \subset \sigma_{\text {ess }}(H)$ доказывается аналогично тому, как это сделано в работе [14].

Пусть $I_{1}, I_{2}$ и $\mathcal{I}$ - единичные операторы в $L_{2}\left(\mathbf{T}^{\nu}\right), L_{2}\left(\left(\mathbf{T}^{\nu}\right)^{2}\right)$ и $L_{2}^{(4)}\left(\mathbf{T}^{\nu}\right)$ соответственно.

Сперва докажем, что $\sigma_{\text {two }}\left(H_{1}\right) \subset \sigma_{\text {ess }}(H)$. Для этого удобно воспользоваться критерием Вейля, т. е. достаточно построить последовательность ортонормированных векторов $\left\{f_{n}\right\} \subset L_{2}\left(\left(\mathbf{T}^{\nu}\right)^{2}\right)$, для которых $\left\|\left(H-z_{0} I_{2}\right) f_{n}\right\| \rightarrow 0$ при $n \rightarrow \infty$. Здесь и далее символ $\|\cdot\|$ означает норму в соответствующих пространствах. Пусть $z_{0} \in$ $\sigma_{\text {two }}\left(H_{1}\right)$ - произвольная точка. Тогда по лемме 3.1 существует точка $p_{0} \in \mathbf{T}^{\nu}$ такая, что $z_{0} \in \sigma_{\text {disc }}\left(h_{1}\left(p_{0}\right)\right)$. Следовательно, существует ненулевая функция $\psi \in L_{2}\left(\mathbf{T}^{\nu}\right)$ такая, что

$$
\left(h_{1}\left(p_{0}\right)-z_{0} I_{1}\right) \psi=0
$$

Положим

$$
f_{n}(p, q)=\frac{\chi_{V_{n}}(p)}{\sqrt{\mu\left(V_{n}\left(p_{0}\right)\right)}} \frac{\psi(q)}{\|\psi\|}
$$

где $\chi_{V_{n}}(\cdot)$ - характеристическая функция множества

$$
V_{n}\left(p_{0}\right)=\left\{p \in \mathbf{T}^{\nu}: \frac{1}{n+1}<\left|p-p_{0}\right|<\frac{1}{n}\right\},
$$

$\mu\left(V_{n}\left(p_{0}\right)\right)$ - лебегова мера множества $V_{n}\left(p_{0}\right)$.

Можно легко проверить, что $\left\{f_{n}\right\}$ является ортонормированной системой. Покажем, что для системы $\left\{f_{n}\right\}$ при $z_{0} \in \sigma_{\text {two }}\left(H_{1}\right)$ верно

$$
\lim _{n \rightarrow+\infty}\left\|\left(H-z_{0} I_{2}\right) f_{n}\right\|=0 .
$$


Заметим, что

$$
\begin{aligned}
\left\|\left(H-z_{0} I_{2}\right) f_{n}\right\|^{2}= & \frac{1}{\|\psi\|^{2}} \int \frac{\chi_{V_{n}}(p)}{\mu\left(V_{n}\left(p_{0}\right)\right)}\left[\int \mid\left(w(p, q)-z_{0}\right) \psi(q)-\right. \\
& \left.-\left.\int v_{1}(q, s) \psi(s) d s\right|^{2} d q\right] d p+\int\left|\int \frac{\chi_{V_{n}}(s) v_{2}(p, s)}{\sqrt{\mu\left(V_{n}\left(p_{0}\right)\right)}} d s\right|^{2} d p .
\end{aligned}
$$

Тогда существует число $C>0$ такое, что второе слагаемое можно оценить через $C \mu\left(V_{n}\left(p_{0}\right)\right)$, которое по построению множества $V_{n}\left(p_{0}\right)$ стремится к нулю при $n \rightarrow+\infty$. А первое слагаемое оценим через

$$
\int \frac{\chi_{V_{n}}(p)}{\mu\left(V_{n}\left(p_{0}\right)\right)}\left\|h_{1}(p)-z_{0} I_{1}\right\|^{2} d p \leqslant \sup _{p \in V_{n}\left(p_{0}\right)}\left\|h_{1}(p)-z_{0} I_{1}\right\|^{2} .
$$

В силу равенства (5.1) оценочное выражение стремится к нулю при $n \rightarrow+\infty$. Таким образом, $z_{0} \in \sigma_{\mathrm{ess}}(H)$. Из произвольности точки $z_{0} \in \sigma_{\mathrm{two}}\left(H_{1}\right)$ вытекает, что $\sigma_{\text {two }}\left(H_{1}\right) \subset \sigma_{\text {ess }}(H)$. Включение $\sigma_{\text {two }}\left(H_{2}\right) \subset \sigma_{\text {ess }}(H)$ доказывается аналогично.

Следовательно, мы доказали, что $\sigma\left(H_{1}\right) \cup \sigma\left(H_{2}\right) \subset \sigma_{\text {ess }}(H)$.

Теперь докажем обратное включение, а именно $\sigma_{\mathrm{ess}}(H) \subset \sigma\left(H_{1}\right) \cup \sigma\left(H_{2}\right)$. В силу компактности оператора $K(z)$ и ограниченности оператора $A^{-1}(z)$ получаем, что $T(z)$ - компактнозначная аналитическая функция в $\mathbf{C} \backslash\left(\sigma\left(H_{1}\right) \cup \sigma\left(H_{2}\right)\right)$. Из самосопряженности оператора $H$ и леммы 4.2 следует, что операторнозначная функция $(\mathcal{I}-T(z))^{-1}$ существует при всех $\operatorname{Im} z \neq 0$. Согласно аналитической теореме Фредгольма (см. книгу [1], теорема XIII.13) заключаем, что операторнозначная функция $(\mathcal{I}-T(z))^{-1}$ существует на $\mathbf{C} \backslash\left(\sigma\left(H_{1}\right) \cup \sigma\left(H_{2}\right)\right)$ всюду за исключением дискретного множества $S$, где она имеет вычеты конечного ранга. Это означает, что $\sigma(H) \backslash\left(\sigma\left(H_{1}\right) \cup \sigma\left(H_{2}\right)\right)$ состоит только из изолированных точек, которые могут иметь предельные точки только в граничных точках множества $\sigma\left(H_{1}\right) \cup \sigma\left(H_{2}\right)$. Отсюда имеем, что $\sigma(H) \backslash\left(\sigma\left(H_{1}\right) \cup \sigma\left(H_{2}\right)\right) \subset \sigma(H) \backslash \sigma_{\text {ess }}(H)$, т. е. $\sigma_{\text {ess }}(H) \subset \sigma\left(H_{1}\right) \cup \sigma\left(H_{2}\right)$.

В силу леммы 3.2 для любого $p \in \mathbf{T}^{\nu}$ оператор $h_{\alpha}(p)$ имеет не более чем по одному простому собственному значению, лежащему левее $m_{\alpha}$ и правее $M_{\alpha}$. Тогда в силу теоремы о спектре разложимых операторов из определения множества $\sigma_{\text {two }}\left(H_{\alpha}\right)$ вытекает, что оно состоит из объединения не более чем двух отрезков, которые расположены в обоих частях множества $\sigma_{\text {three }}(H)$. Следовательно, множество $\sigma_{\mathrm{ess}}(H)$ состоит из объединения не более чем пяти отрезков. Теорема 2.2 полностью доказана.

ДокАЗАТЕЛЬСТво теОРЕмы 2.3. Пусть $\min _{p \in \mathbf{T}^{3}} \Delta_{\alpha}(p ; m) \geqslant 0$. Тогда из леммы 3.3 вытекает, что для любого $p \in \mathbf{T}^{3}$ оператор $h_{\alpha}(p)$ не имеет собственных значений, лежащих левее $m$. В силу определения множества $\sigma_{\text {two }}\left(H_{\alpha}\right)$ имеем, что

$$
\sigma_{\text {two }}(H) \cap(-\infty, m)=\varnothing .
$$

(a) Пусть $\min _{p \in \mathbf{T}^{3}} \Delta_{\alpha}(p ; M) \geqslant 0$. Аналогично можно показать, что

$$
\sigma_{\text {two }}\left(H_{\alpha}\right) \cap(M, \infty)=\varnothing .
$$


Теоремы 2.1 и 2.2 завершают доказательство части (а) теоремы 2.3.

(б) Пусть $\max _{p \in \mathbf{T}^{3}} \Delta_{\alpha}(p ; M)<0$. В силу леммы 3.4 для любого $p \in \mathbf{T}^{3}$ оператор $h_{\alpha}(p)$ имеет единственное простое собственное значение $E_{\alpha}(p)>M$. Так как функции $v_{\alpha i}(\cdot), i=1,2$, и $w_{\alpha}(\cdot, \cdot)$ непрерывны в своих областях определения, то функция $E_{\alpha}(\cdot)$, сопоставляющая элементу $p \in \mathbf{T}^{3}$ собственное значение $E_{\alpha}(p)$, также непрерывна на компактном множестве $\mathbf{T}^{3}$. Отсюда вытекает, что множество значений $\operatorname{Im} E_{\alpha}$ функции $E_{\alpha}(\cdot)$ есть отрезок $\operatorname{Im} E_{\alpha}=\left[c_{\alpha} ; d_{\alpha}\right]$, где $c_{\alpha}>M$. Из определения множества $\sigma_{\text {two }}\left(H_{\alpha}\right)$ следует, что

$$
\sigma_{\text {two }}\left(H_{\alpha}\right) \cap(M, \infty)=\left[c_{\alpha} ; d_{\alpha}\right], \quad c_{\alpha}>M .
$$

Учитывая теоремы 2.1 и 2.2, получим доказательство части (б) теоремы 2.3.

Остальные утверждения теоремы 2.3 доказываются аналогично. Теорема доказана.

Благодарности. Автор выражает глубокую благодарность Г. М. Жиислину и рецензенту за ценные и полезные замечания. Работа поддержана Deutsche Forschungsgemeinschaft (DFG) (грант № TR368/6-1).

\section{Список литературы}

[1] М. Рид, Б. Саймон, Методъ современной математической физики, т. 4: Анализ операторов, Мир, М., 1982.

[2] Г. М. Жислин, Труды ММО, 9, 1960, 81-120.

[3] W. Hunziker, Helv. Phys. Acta, 39 (1966), 451-462.

[4] С. Н. Лакаев, М.Э. Муминов, ТМФ, 135:3 (2003), 478-503.

[5] S. Albeverio, S. N. Lakaev, Z. I. Muminov, Math. Nachr., 280:7 (2007), 699-716, arXiv: math-ph/0312050.

[6] S. Albeverio, S. N. Lakaev, Z. I. Muminov, Russ. J. Math. Phys., 14:4 (2007), 377-387.

[7] S. Albeverio, S. N. Lakaev, R. Kh. Djumanova, Rep. Math. Phys., 63:3 (2009), 359-380.

[8] В. Хейне, М. Коэн, Д. Уэйр, Теория псевдопотенциала, Мир, М., 1973.

[9] Т. Х. Расулов, ТМФ, 163:1 (2010), 34-44.

[10] R. L. Hall, J. Phys. A, 39:4 (2006), 903-912, arXiv: math-ph/0512079.

[11] K. Chadan, R. Kobayashi, J. Phys. A, 38:5 (2005), 1133-1145, arXiv: math-ph/0409018.

[12] Ж. И. Абдуллаев, Узб. матем. журн., 1996, № 1, 3-10.

[13] Т. Х. Расулов, Изв. вузов. Матем., 2008, № 12, 59-69.

[14] Ю. Х. Эшкабилов, ТМФ, 149:2 (2006), 228-243.

Поступила в редакцию 16.05.2010, после доработки 16.06.2010 